\title{
A Novel Device for the Measurement of the Mechanical and Magnetic Axes of Superconducting Magnet Assemblies for Accelerators
}

\author{
J. Garcia-Perez, S. Aznar, J. Billan, F. Fischer, P. Galbraith, S. Goy, N. Mermillod, G. Peiro, G. Patti, and C. Rathjen
}

\begin{abstract}
In the context of the LHC superconducting magnet production, especially for dipoles and quadrupoles due to their complexity, it is foreseen to perform acceptance tests, at an early production stage, to detect possible significant deviations from the design values. The knowledge of the magnetic field geometry is very important, especially for the main magnets. In order to get this information a new device has been conceived that measures the magnets at room temperature during different stages of construction. This device incorporates a sensitive measuring probe and an efficient data acquisition system because the coils are only powered at about $10^{-5}$ of the nominal D.C. current. It is dedicated to Quadrupole and Dipole [by using Quadrupole-Configured Dipole (QCD) transformation] magnets, but is also easily adaptable to higher order magnets ( $n=3,4$ and 5 ) by specific orientation of the search coils. It is equipped with magnetic sensors (4 fixed tangential coils and $\mathrm{AC}$ excitation current for the magnet) and position sensors (3D-laser tracker and light reflector) that allow the simultaneous detection of the magnetic field axis and the cold bore axis.

It is equipped as well with a set of 4 LEDs and associated with a CCD camera that allows both the measurement of the cold bore diameter and its position with respect to the mole. This paper describes the system and reports the first results measured on the pre-series magnets recently assembled.
\end{abstract}

Index Terms-Laser tracker, LED's application, LHC magnets, magnetic axis, QCD magnet configuration.

\section{INTRODUCTION}

$\mathbf{T}$ HE LHC machine is very sensitive to magnet misalignment and special attention has been paid to develop dedicated instruments to ensure good alignment. Nearly all the magnets are superconducting, and therefore not accessible inside their cryostats. Therefore very precise measurements are necessary during production in view of possible adjustments of their geometry. Measurement accuracy within $\pm 0.1 \mathrm{~mm}$ must be guaranteed. A first major decision was to buy 3-D laser tracker devices to facilitate certain very delicate operations in the magnet assembly process. Taking advantage of the possibilities given by these devices, a mole has been designed to measure the mechanical axis of the CBT's of the magnets. This operation is particularly important for dipole magnets which are $15-\mathrm{m}$ long and curved with a 10-mm sagitta. We

Manuscript received September 24, 2001.

J. Garcia-Perez, S. Aznar, J. Billan, F. Fischer, P. Galbraith, S. Goy, G Peiro, and C. Rathjen are with the CERN/LHC, in the European Organization for Nuclear Research (CERN), 1211 Geneva 23, Switzerland (e-mail: Juan.Garcia.Perez@cern.ch).

N. Mermillod and G. Patti are with the CERN/EST Division, in the European Organization for Nuclear Research (CERN), 1211 Geneva 23, Switzerland.

Publisher Item Identifier S 1051-8223(02)04276-8. studied the idea to incorporate in such a mole the capability to measure the magnetic axis at the same time.

The two geometry parameters of the magnetic field to be measured are the magnetic axis position with respect to intermediate fiducials, expressed in $\mathrm{mm}$, and the field direction with respect to the vertical, expressed in mrad. Both can be determined from magnetic measurements performed with rotating search coils and harmonic analysis. In this measurement, for the field direction, a gravity sensor and an encoder allow for accuracy better than $\pm 0.1 \mathrm{mrad}$, but for the magnetic axis, a complicated system is required to refer the measurement to the cold mass fiducials. On the contrary, the system described in the following, using fixed coils can easily measure the position of the magnetic axis and relate it to the fiducials thanks to the 3-D laser tracker. It can also give the field direction, but with accuracy limited to $\pm 1 \mathrm{mrad}$.

\section{GENERAL DESCRIPTION}

The mole has been designed with the aim to measure in one operation the geometry of the magnetic field and the CBT with respect to an external reference system, with the aid of a 3-D Laser Tracker [1]. The mole, as shown in Fig. 1, has a mechanically defined center where the three main sensors are precisely placed with in a common cross-section plane to avoid any parallax errors:

1) A corner cube reflector centered in the mole

2) Four fixed tangential search coils

3) Four LEDs

The mole can travel inside the CBT and be positioned at fixed points. It is oriented with respect to gravity by a motor. To know its position in space, a Laser Tracker is used. This device is a portable 3-D measuring system, having a precision better than $10 \mathrm{ppm}$ of the measured distance (at $2 \sigma$ ). It sends a laser beam onto a Corner Cube Reflector placed at the point to be measured. The head of the Laser Tracker, source of the beam, is then oriented until the laser is reflected back to a detector. In our case, the corner cube is replaced by a prism drawn in a cylindrical piece of glass that is collinear with the axis of the mole, with its center placed at the intersection of the symmetry planes of the four tangential coils. For this purpose the laser has to travel inside the CBT, so it is important not to have temperature gradients that could perturb straight light propagation. This is the reason to have minimum heating sources on the mole. To know the position with respect to the CBT the LEDs and a CCD camera installed inside the mole are used. The LED's project 


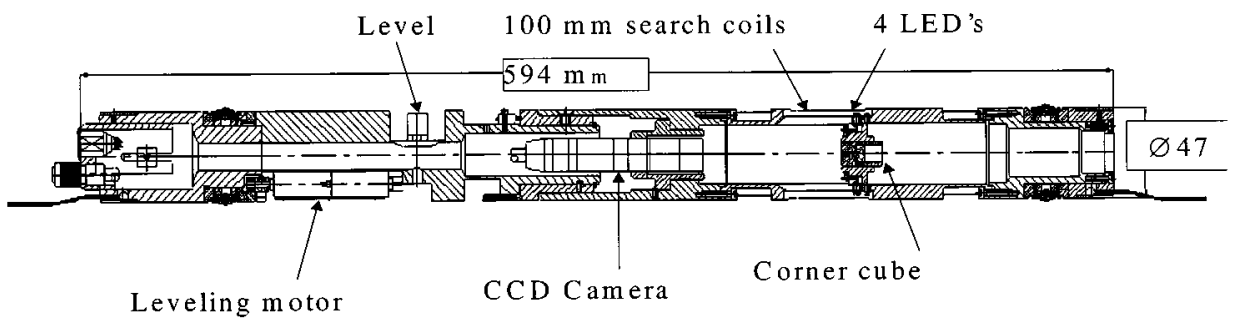

Fig. 1. Mole to measure mechanical and magnetic axis of LHC superconducting magnets at room temperature.

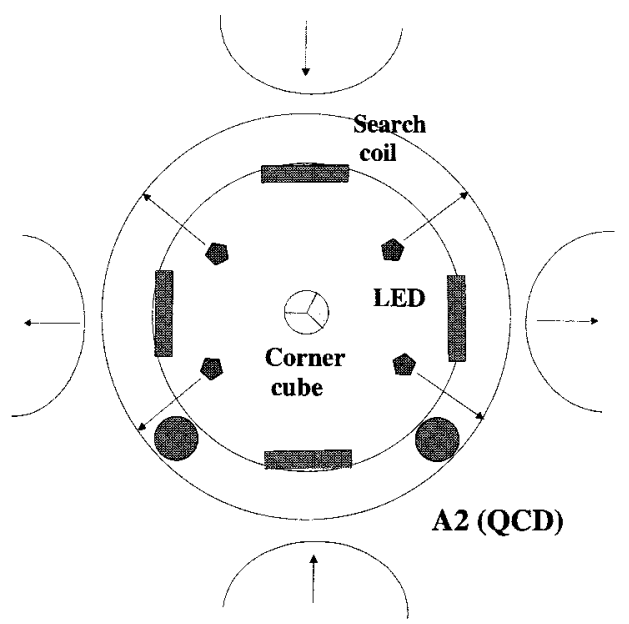

Fig. 2. Cross section of the mole inside a quadrupole configured dipole.

light spots on to the CBT and the image of the light spots is analyzed by an image processing system. The four tangential search coils then measure the magnetic axis [2]. The magnet is powered with small $\mathrm{AC}$ current and the voltages induced in the coils are synchronously detected. For a dipole, we excite the two poles in opposition instead of in series then generating a skew quadrupole field, the so-called "Quadrupole-Configured Dipole" (QCD). See Fig. 2.

The coil support structure of the mole can be rotated around its axis to configure it in the best orientation accordingly to the magnet type to be measured ( $n=1,2,3,4,5)$.

Labview ${ }^{\mathrm{TM}}$ software is used to control the motors and the data acquisition of the level meter and search coils signals. AXYZ ${ }^{\mathrm{TM}}$ software from Leica is used for the Laser Tracker.

\section{Mole Positioning System}

The mole is attached to traction cables at both ends. Motorized pulleys pull them. A Laser Tracker measures the longitudinal position along the magnet beam tube. This is done with a precision of $0.1-\mathrm{mm}$. Once in position another small motor, placed inside the mole, rotates the mole to the vertical position with the help of a level meter. The accuracy is better than $1 \mathrm{mrad}$.

\section{Measuring Mole Position}

The 3-D Laser Tracker measures the position of the mole with the aid of a corner cube reflector in a general reference system associated to the magnet. It is also necessary to measure the mole position with respect to the cold bore. This is done by analyzing the image of the diffuse reflections of the four LEDs on
Cold bore

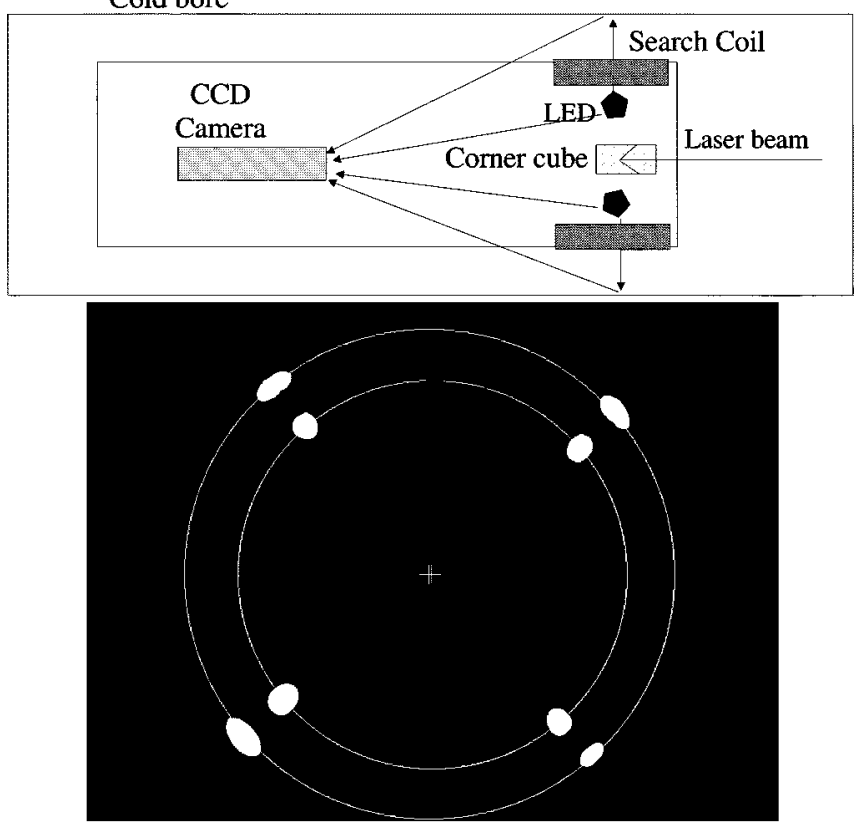

Fig. 3. Sketch of the subsystem to measure the mole position with respect to CBT. The LEDs, coil's center of gravity and retroreflector center are in the same plane. The lower figure shows the resulting image after filtering. The inner fitted circle is obtained from the light coming directly from the LEDs through a hole made in the Led support piece used as additional reference information. The two center crosses represent the two fitted circle centers.

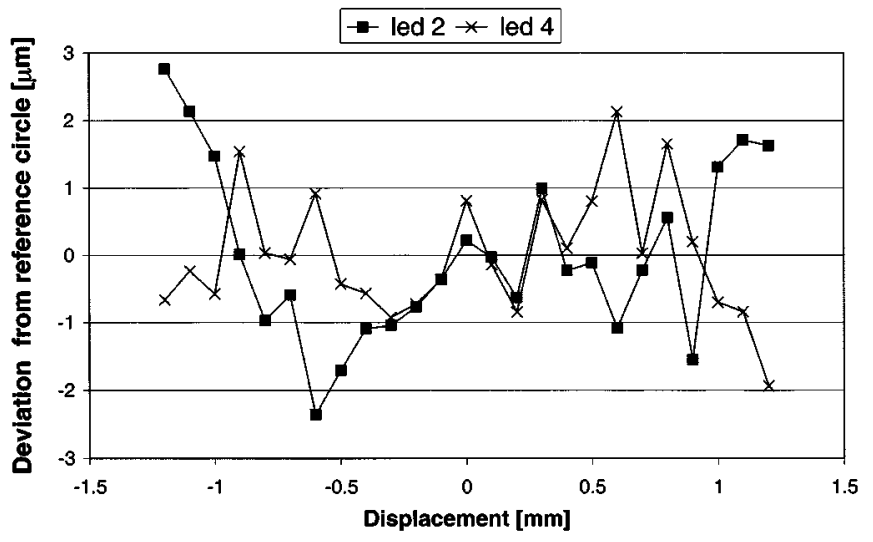

Fig. 4. Residuals from the LED's spot positions in reference tube of 50-mm diameter versus lateral displacement of the mole inside the reference tube.

the CBT. (See Fig. 3). The four spots of the LEDs can be fitted to a circle from which the center is determined in the local reference system of the mole. The four residuals (difference between the center of gravity of reflected spots and the positions fitted to obtain a circle) give the tube cross-section at this point. 


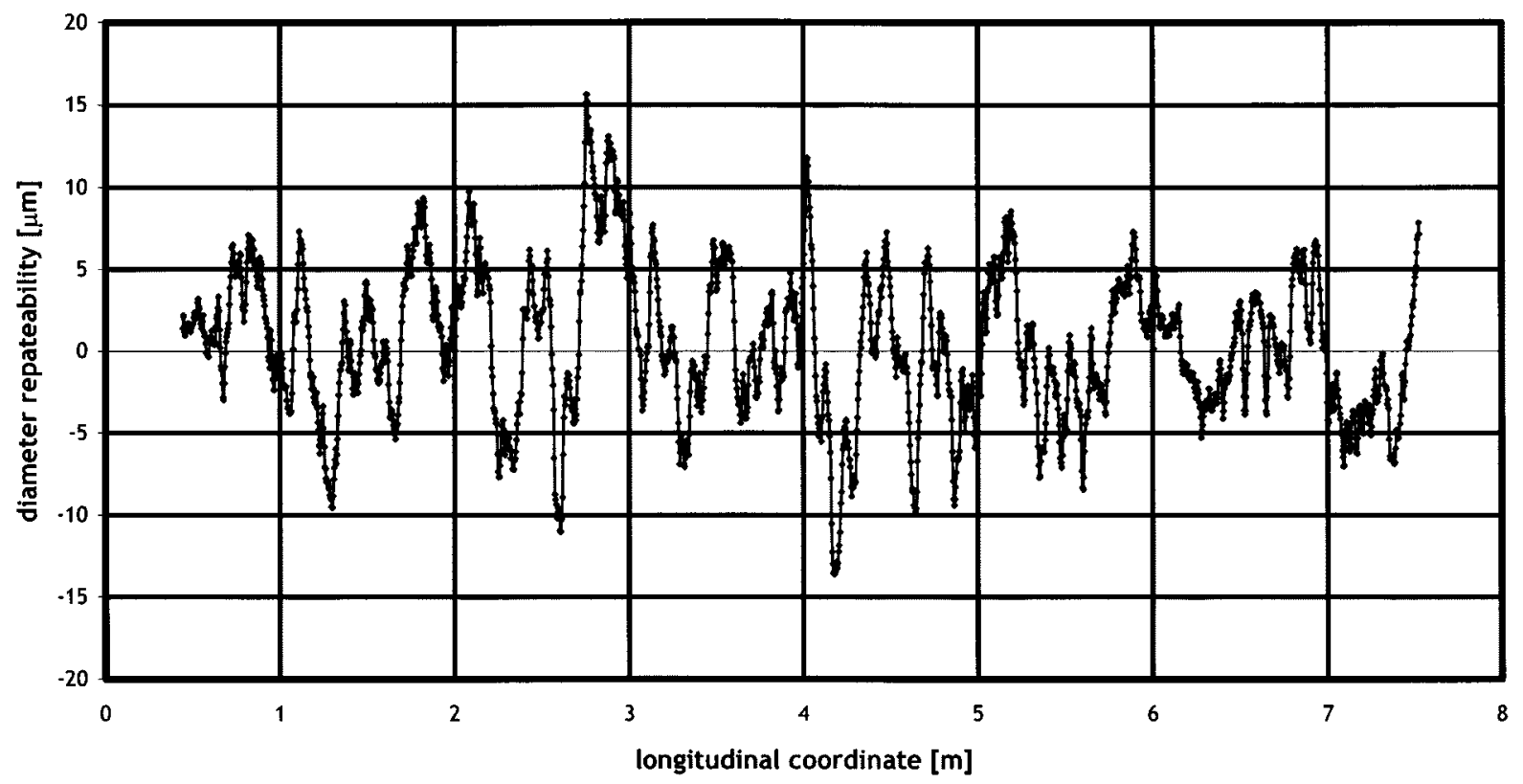

Fig. 5. Repeatability of the diameter measurement of a CBT using LED spots positions.

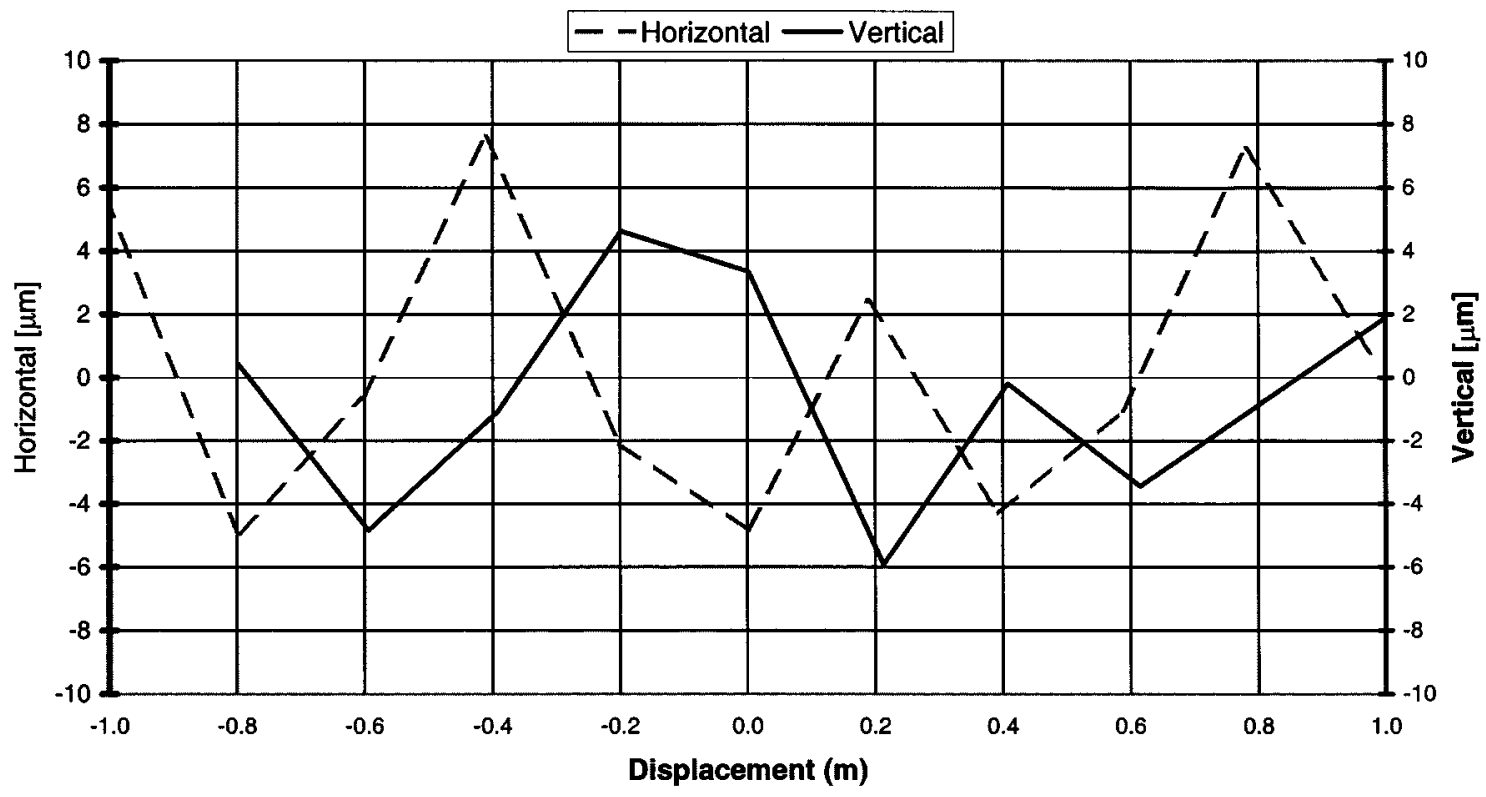

Fig. 6. Residuals of the sextupole corrector magnet calibration.

TABLE I

Sensitivity of THE System at 10E-4 TO 10E-6 OF THE MAXIMUM LHC QUADRUPOLE FIELD

\begin{tabular}{|c|c|c|c|}
\hline $\begin{array}{c}\text { Coil Voltage } \\
(\mathbf{m V})\end{array}$ & $\begin{array}{c}\text { Input Current } \\
(\mathbf{m A})\end{array}$ & $\begin{array}{c}\text { Field at } \mathbf{r = 1 7} \mathbf{~ m m} \\
(\mathbf{T})\end{array}$ & $\begin{array}{c}\text { Sensitivity } \\
(\mu \mathbf{m})\end{array}$ \\
\hline 37 & 100 & $3.10-4$ & $<1$ \\
\hline 3.7 & 10 & $3.10-5$ & 2 \\
\hline 0.37 & 1 & $3.10-6$ & 10 \\
\hline
\end{tabular}

Fig. 4 shows the performance of the CCD-LED system measuring the inner diameter of a calibrated, 50-mm diameter tube when it is off axis. The curves show, for the two horizontal LEDs, the measured deviations residuals from a perfect $50-\mathrm{mm}$ circle. They are in the order of few microns. The center of the fitted circle gives the relative position between the mole and the tube. The standard deviation of the errors of the measurements of the $0.1-\mathrm{mm}$ displacements is 1 micron.

The Fig. 5 shows repeatability results of two diameter measurements in a test CBT. The longitudinal positions for the two passes agree within $1 \mathrm{~mm}$. The standard deviation of this distribution is $5 \mu \mathrm{m}$.

\section{Measuring Mechanical and Magnetic Axes}

The 3-D measurement of the mole's corner cube reflector coordinates by the Laser Tracker at the different positions of the CBT gives the mechanical axis of the beam tube, after correction of the off centered position of the mole measured by LED-CCD. 


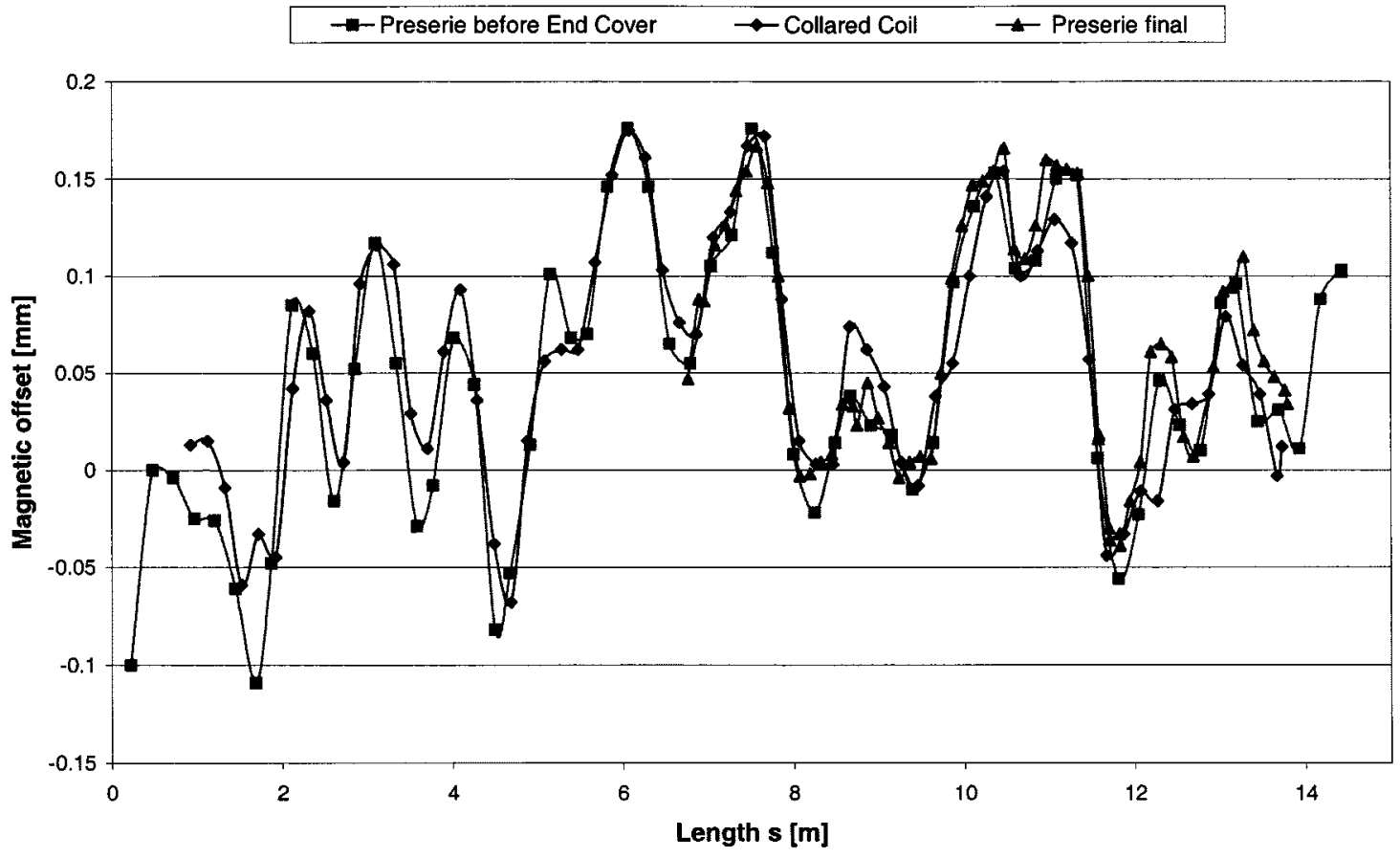

Fig. 7. Offsets measured by the mole at three different stages of the magnet assembly.

To obtain the magnetic axis of the magnet one has to add to this mechanical axis the magnetic offset measured by the search coils.

Typically an AC $(25 \mathrm{~Hz})$ current of $0.5 \mathrm{~A}$ is injected into the magnets. The length of the search coils is $10-\mathrm{cm}$ for an approximately 0.6-m2 magnetic surface area. Table I shows the high sensitivity of the mole.

Fig. 6 shows the residuals of the sextupole calibration. To find the magnetic axis of this calibration magnet the mole has to be used with two probe orientations. For the vertical component the angle is 0 (like for the dipole single probe orientation) and 45 degrees for the horizontal component. In order to avoid too much mixing between horizontal and vertical components, the mole has to be oriented to its nominal position better than 5 degrees for any magnet type. The level meter gives the orientation to be able to correct for this.

\section{Periodic Calibration of the Mole}

The device needs periodic verification of the calibration to check for stability. A calibration system composed of a calibrated 50-mm tube mounted on an $X-Y$ translation table has been produced to be used with the Laser Tracker. By turning the mole inside this system by $180^{\circ}$ one can detect the mechanical offset of the corner cube with respect to the mole center as well as any misalignment of the subsystem LED-CCD camera. It is foreseen to make a calibration every month in the beginning and then less frequently if the mole is stable.

\section{RESULTS}

The mole has been used to measure several dipole magnets with their correctors and two quadrupoles. Fig. 7 shows the ver-
TABLE II

ShIFT OF THE Mechanical AND THE MAGNETIC AXES

\begin{tabular}{|c|c|c|c|c|c|c|c|c|}
\hline & \multicolumn{8}{|c|}{ Mechanical-Magnetic axis[mm] } \\
\hline & \multicolumn{4}{|c|}{ Internal Tube } & \multicolumn{4}{|c|}{ External Tube } \\
\hline & \multicolumn{2}{|c|}{ Horizontal } & \multicolumn{2}{|c|}{ Vertical } & \multicolumn{2}{|c|}{ Horizontal } & \multicolumn{2}{|c|}{ Vertical } \\
\hline & Ave & $\sigma$ & Ave & $\sigma$ & Ave & $\sigma$ & Ave & $\sigma$ \\
\hline P2A2 & 0.09 & 0.03 & & 0.02 & $\overline{05}$ & 0.03 & -0.08 & 0.03 \\
\hline$P 20$ & 25 & 0.00 & & & 0.20 & 00 & 0.01 & 0.6 \\
\hline & & & & & & & & 0.6 \\
\hline PSOI & 16 & 0.05 & $=0$ & 0.6 & 0.20 & .04 & -0.05 & 0.02 \\
\hline Global & 0.12 & 0.05 & -0.04 & 0.02 & 0.09 & 0.06 & -0.04 & 0.04 \\
\hline
\end{tabular}

tical offsets between the CBT and the magnetic axis measured at three different stages of the magnet assembly: first at collared coil, then before end covers mounting and later after cold mass completion (over half-length only). The magnet is the first of the pre-series. One can note the very good agreement between the measurements in spite of the fact that experimental points are not taken at exactly the same longitudinal position. One can also note a periodicity along the length that is not yet understood.

In Table II we show the shifts between the mechanical and magnetic axis measured in four dipoles [3]. The $\sigma$ is calculated with the differences between the mechanical and magnetic axis. The global shift is computed as the quadratic sum of the individual rms divided by the square root of the dipole number. For first MBP2O1 and first pre-series (PSO1) magnets one can note significant deviations bigger than $3 \sigma$ in both apertures on the horizontal coordinate.

Fig. 8 shows the results of the N1 pre-series dipole. As it can be seen, both apertures are within the tolerance for mechanical and magnetic axis. Nevertheless a systematic shift can be seen 

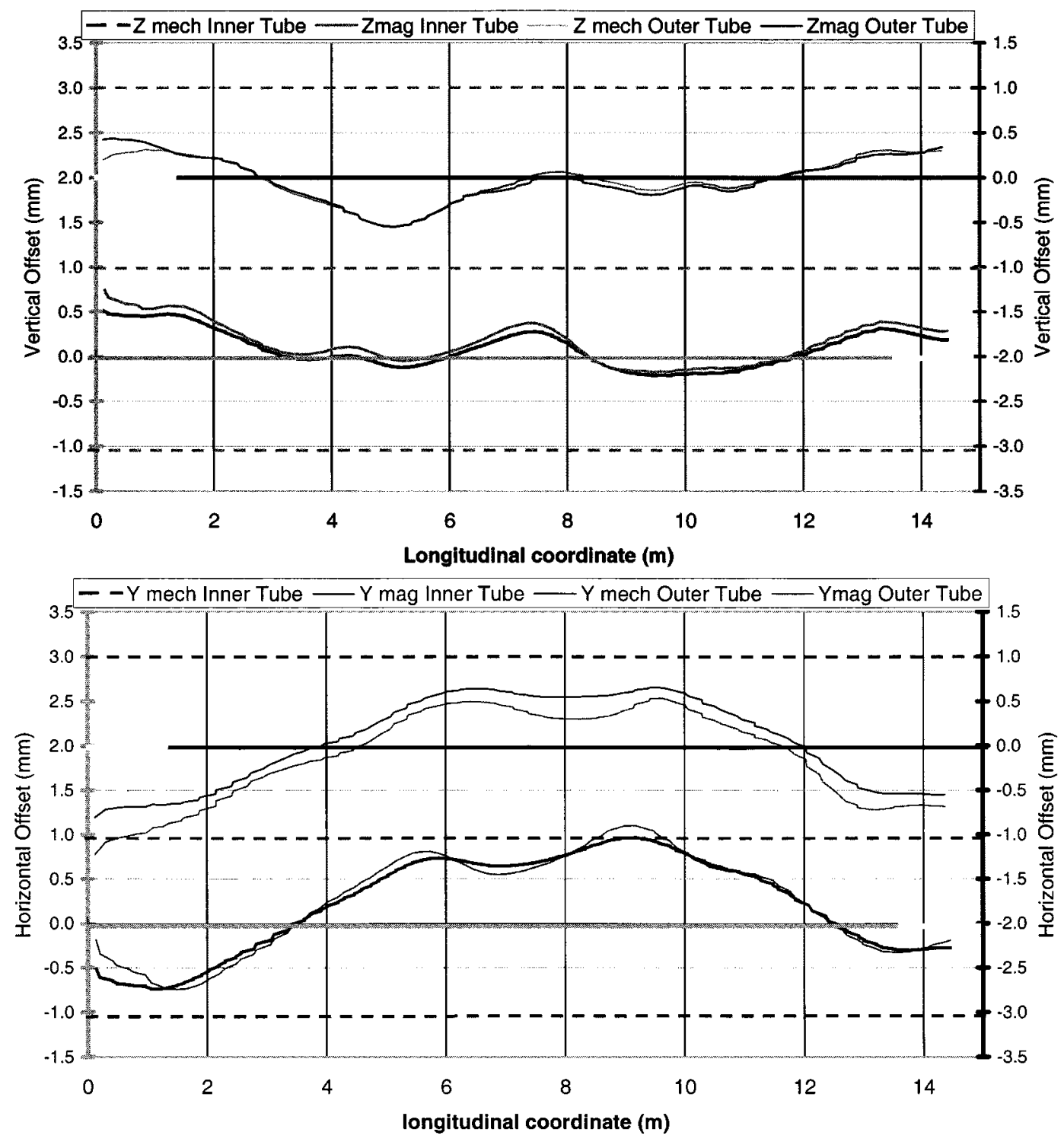

Fig. 8. Results of the measurement of the first pre-series N1 dipole. The offsets between the mechanical and magnetic axis measurements and the theoretical axis for both apertures are plotted. The upper plot shows the vertical offsets and the lower the horizontal. The dashed lines represent the tolerance radius of $1 \mathrm{~mm}$.

in the horizontal component of the outer tube between magnetic and mechanical axis.

\section{CONCLUSIONS}

We have described a device to measure both the mechanical and the magnetic axes of the LHC dipoles at the same time. The system operates in combination with a Laser Tracker device. The local accuracy is $0.03 \mathrm{~mm}$ to be added to the $10 \mathrm{ppm}$ of the Laser Tracker. The system can be used to measure the axis of any type of LHC magnet by configuring the angular position of the search coils. The angular position inside the CBT is measured by a level meter and controlled by a motor to better than $1 \mathrm{mrad}$.

It shows excellent performance, good sensitivity and is easy to use.

The results, in the few magnets measured, are good apart from some small systematic shifts in one of the dipole prototypes and one pre-series.

\section{ACKNOWLEDGMENT}

The authors would like to thank H. Reymond who worked on the electronics and data acquisition and R. Beltron, L. Gaborit, F. Girod, and A. Musso who produced the coils, performed their calibration and mounting in the mole. Also they would like to thank M. Bajko, A. Pardons, and P. Gillieron for their help in magnetic axis measurements.

\section{REFERENCES}

[1] J. Billan, F. Fischer, P. Galbraith, J. Garcia, N. Mermillod, G. Patti, and C. Rathjen, "Development of techniques to measure the magnetic field geometry of LHC magnets," in IMMW11, Brookhaven, USA, 1999.

[2] J. Billan, F. Fischer, P. Galbraith, J. Garcia, N. Mermillod, and G. Patti, "An AC field static system for measuring the magnetic axis of LHC superconducting magnets in warm condition," in IMMW11, Brookhaven, USA, 1999.

[3] M. Bajko, J. Billan, M. Buzio, G. Deferne, P. Ferracin, J. Garcia-Perez, W. Scandale, and E. Todesco, "Geometric and magnetic axes of the LHC dipole," in PAC, Chicago, 2001. 